\title{
Micellization and Intermicellar Interactions in Aqueous Sodium Dodecyl Benzene Sulfonate Solutions
}

\author{
DANIEL C. H. CHENG AND ERDOGAN GULARI ${ }^{1}$ \\ Department of Chemical Engineering, University of Michigan, Ann Arbor, Michigan 48109
}

Received December 3, 1981; accepted March 12, 1982

\begin{abstract}
Quasielastic light scattering was used to investigate the size and shape of sodium dodecyl benzene sulfonate (SDBS) micelles in aqueous solutions. Measurements were made as a function of temperature and $\mathrm{NaCl}$ and SDBS concentrations. Light scattering data indicate that the micelles are spherocylinders with a semiminor axis of $22 \AA$. The length of the micelles increases strongly with salt concentration and decreases with temperature. The minimum micelle has an aggregation number of $n^{0}=28$ and a hydrodynamic radius of $R=22 \AA$. Diffusion coefficient and intensity data were analyzed using a thermodynamic theory of micellar aggregation and a model based on DLVO theory of interactions.
\end{abstract}

\section{INTRODUCTION}

Due to their importance in tertiary oil recovery, surfactants of the alkylaryl sulfonate family have received considerable attention in recent years. In this paper we report the results of our quasi-elastic light-scattering measurements performed on dilute aqueous solutions of sodium dodecyl benzene sulfonate (SDBS).

Many investigators have performed static (1-2) and dynamic (3-7) light-scattering experiments on aqueous solutions of ionic micelles. These results indicate that large, polydisperse micellar aggregates are present, and that the size of the aggregates depends on temperature, ionic strength, and in some cases the counterion. Despite extensive experimental and theoretical work, our understanding of micellar solutions is not complete. This is due to the fact that the size and shape of the micelles may be changed by changing either the amphiphile or the electrolyte concentration or the temperature of the solution. Thus one has to rely on theoretical models and several different experi-

\footnotetext{
'Author to whom correspondence should be addressed.
}

mental techniques to separate the effects of intermicellar interactions on the equilibrium and transport properties from those due to a change in micelle size and shape. We present extensive data on the size and shape of SDBS micelles and analyze our results in terms of two different models.

\section{EXPERIMENTAL}

\section{A. Materials and Sample Preparation}

The SDBS $(\mathrm{MW}=348.5)$ used in this study was Siponate DS-10 sold by Alcolac Company and claimed to be $98 \%$ active. This was further purified by successive extraction and recrystallization from isopropyl and methyl alcohols. We estimate the purity to be about $99.5 \%$. An infrared spectroscopic isomeric purity check indicated that the surfactant was a mixture of $80 \%$ para and $20 \%$ ortho isomers with no detectable meta isomer. Water was doubly distilled and deionized and the $\mathrm{NaCl}$ was of ACS reagent grade.

Micellar solutions were prepared by first dissolving a weighed amount of $\mathrm{NaCl}$ in water and adding purified SDBS to the warm $\mathrm{NaCl}$ solution afterward. Solutions were stored $\sim 5-10^{\circ} \mathrm{C}$ above their critical micellar 
temperatures in stoppered flasks for 12 hours in order to allow for complete dissolution before the final dilution to the desired concentrations.

The samples used for light-scattering measurements were filtered through $0.2-\mu \mathrm{m}$ Millipore filters to remove the dust.

\section{B. Critical Micellar Temperatures}

The critical micellar temperatures (CMT) were determined as the midpoint of a small temperature range over which the hydrated solid phase of SDBS clarified and dissolved completely upon slow heating of the sample. These temperatures were checked by starting at a temperature above the CMT and slowly reducing the temperature until the hydrated SDBS phase started forming. CMT values determined by both methods agreed to within $\pm 0.8^{\circ} \mathrm{C}$.

\section{Light-Scattering Measurements}

Integrated intensity and dynamic lightscattering measurements were made with a spectrometer described previously (8). A 2 W stabilized Argon-ion laser was the light source $(\lambda=514.5 \mathrm{~nm})$ and the autocorrelation functions were measured with a 64channel Malvern multibit correlator. Some correlation functions were obtained by using software dust discrimination in order to completely eliminate distortions due to occasional dust. The sample temperature was controlled to $\pm 0.1^{\circ} \mathrm{C}$ and at least four hours were allowed for thermal equilibration.

\section{DATA ANALYSIS}

\section{A. Apparent Molecular Weights}

The apparent molecular weights $M$ were calculated from

$$
\frac{H C}{R_{\mathrm{v}}}=\frac{1}{M}\left(1+q^{2} R_{g}^{2} / 3\right)
$$

where $H=4 \pi^{2} n_{0}^{2}(\partial n / \partial C)^{2} / N_{\mathrm{A}} \lambda_{0}^{4}$, with $n_{0}, C$, $N_{\mathrm{A}}$, and $\lambda_{0}$ being the refractive index of the solution, concentration of the surfactant,
Avogadro's number, and the wavelength of the incident light, respectively. The Rayleigh ratio for vertically polarized light, $R_{v}$, was obtained by using benzene as a reference, $q$ $=4 \pi n \sin (\theta / 2) / \lambda$ is the scattering vector, and $R_{\mathrm{g}}$ is the radius of gyration. Measurements of the scattered intensity were made over a range of angles $\left(30^{\circ} \leq \theta \leq 150^{\circ}\right)$ and by extrapolation to zero angle, the effect of the $q^{2} R_{\mathrm{g}}^{2} / 3$ term in Eq. [1] was eliminated.

\section{B. The Apparent Radii of Gyration}

The radii of gyration were determined from the angular dissymmetry of the scattered light using

$$
\frac{I(0)}{I(q)} \cong\left(1+q^{2} R_{\mathrm{g}}^{2} / 3\right)
$$

For some of our solutions the dissymmetry was too small to measure the $R_{\mathrm{g}}$ values reliably.

\section{The Apparent Diffusion Coefficients and Hydrodynamic Radii}

For a monodisperse solution of small noninteracting particles the homodyne autocorrelation function of the scattered light is a single exponential with a time constant $\tau_{c}$ $=1 / 2 D q^{2}$, where $D$ is the diffusion coefficient of the particles. For a polydisperse (and possibly interacting) solution the homodyne autocorrelation function deviates from a single exponential and its deviation contains information about the polydispersity of the sample and in some cases about the shape of the scattering particle.

The correlation functions were analyzed by a modified method of cumulants (9) to determine the $Z$-average diffusion coefficient $\bar{D}$ and the relative variance $V$ of the scatterers. In all of our analysis we have ignored the contribution from the rotational motion of the micelles, since in the worst case this accounted for less than $2 \%$ of the scattered intensity.

The $Z$-average apparent hydrodynamic radii $\bar{R}_{\mathrm{h}}$ were calculated from the diffusion 
coefficient using the Stokes-Einstein relationship

$$
\bar{D}=k_{\mathrm{B}} T / 6 \pi \eta \bar{R}_{\mathrm{h}},
$$

where $\eta$ is the solvent viscosity and $k_{\mathrm{B}}$ is the Boltzmann constant.

\section{INTERPRETATION OF THE DATA}

Formation and growth of SDBS micelles in aqueous solutions is a function of temperature, and $\mathrm{NaCl}$ and SDBS concentration. To elucidate the effect of each variable independently we have made two types of measurements; (i) holding SDBS and $\mathrm{NaCl}$ concentrations constant while varying the temperature and (ii) holding $\mathrm{NaCl}$ concentration and the temperature constant while varying the SDBS concentration. The first set of data was analyzed by a thermodynamic model. The second set was analyzed using the DLVO theory of colloidal interactions. Each method of analysis is briefly described below.

\section{A. Thermodynamic Analysis}

A thermodynamic model similar to the one used by Mazer et al. (10) and Overbeek and Tausk (11) was used to interpret the variable-temperature data. This model is a modified version of the equal probability stepwise association model. The basic assumptions are: (i) there are no aggregates smaller than the minimum spherical micelle with an aggregation number $n_{0}$ (=28 for SDBS), and (ii) the larger micelles with aggregation number $n>n_{0}$ are spherocylinders (a shape consistent with our experimental observations as will be seen later), (iii) $X$, the total solute mole fraction, is much less than one (dilute solution), and (iv) $X \gg X_{1}$, where $X_{1}$ is the monomer concentration.

The results of this model are (10)

$$
\begin{gathered}
X_{n}=\left(B X_{1}\right)^{n} / K, \\
B=\exp \left[\left(\mu_{1}^{0}-\mu^{0}\right) / k_{\mathrm{B}} T\right], \\
K=\exp \left[\left(\mu_{n_{0}}^{0}-n_{0} \mu^{0}\right) / k_{\mathrm{B}} T\right],
\end{gathered}
$$

and

$$
K X=\left(B X_{1}\right)^{n_{0}}\left(\frac{n_{0}}{1-B X_{1}}+\frac{B X_{1}}{\left(1-B X_{1}\right)^{2}}\right)
$$

where $X_{n}$ is the mole fraction of a micelle with an aggregation number $n, \mu_{1}^{0}$ is the standard chemical potential of the monomer, $\mu_{n_{0}}^{0}$ is the standard chemical potential of the minimum spherical micelle, and $\mu^{0}$ is the standard chemical potential of a monomer in the cylindrical portion of the micelle.

Combining Eq. [7] with Eq. [4] one can then obtain the mole fractions $X_{n}$ as a function of the parameter $K X$. Once we know $X_{n}$ we can readily calculate the number- and weight-averaged aggregation number $\bar{n}_{\mathrm{n}}$ and $\bar{n}_{\mathrm{w}}$ and the relative variances $V$. Since the primary quantity in a quasi-elastic light-scattering experiment is the diffusion coefficient we also calculated the $Z$-average diffusion coefficient $\bar{D}$ by

$$
\bar{D}=\sum G_{i} D_{i},
$$

where $G_{i}$ is the fraction of light scattered by the $i$ th species with a diffusion coefficient $D_{i}$ (calculated from Perrin's equations for prolate ellipsoids of revolution); $\bar{R}_{\mathrm{h}}$ is obtained from $\bar{D}$ using the Stokes-Einstein relationship Eq. [3]. Relative variances of $\bar{D}$ and $\bar{R}_{\mathrm{h}}$ are also calculated in a similar fashion.

\section{B. Intermicellar Interaction Potential Analysis}

To the first order in volume fraction $\phi$ the apparent diffusion coefficient $D$ and the apparent molecular weight $M$ are related to their true values $D_{0}$ and $M_{0}$ by

$$
\begin{gathered}
D=D_{0}\left(1+k_{\mathrm{D}}^{\prime} \phi\right), \\
M^{-1}=M_{0}^{-1}\left(1+k_{\mathrm{I}}^{\prime} \phi\right),
\end{gathered}
$$

where the coefficient $k_{\mathrm{I}}^{\prime}$ is proportional to the second virial coefficient and $k_{\mathrm{D}}^{\prime}$ is a measure of the intermicellar interactions on $D$. Applications of Eqs. [9] and [10] to macromolecular solutions are straightforward but their use for micellar solutions necessitate the 
extra assumption that the micellar size does not change with the volume fraction $\phi$ (or the concentration $C$ ) of the micelles. Since there are both repulsive (electrostatic) and attractive (London-van der Waals) forces between the micelles we can expect $k_{\mathrm{I}}^{\prime}$ and $k_{\mathrm{D}}^{\prime}$ to change both in sign and magnitude as one varies the salt concentration of micellar solutions.

For rigid spherical particles $k_{\mathrm{I}}^{\prime}$ is related to the pair interaction potential $V(x)$ by (12)

$$
\begin{aligned}
k_{\mathrm{I}}^{\prime}=8+24 \int_{0}^{\infty}(1+x)^{2} \\
\\
\times\left[1-e^{-v(x) / k_{\mathrm{B}} T}\right] d x,
\end{aligned}
$$

where $x=(R-2 a) / 2 a, R$ is the distance between the centers of the two particles, and $a$ is the radius of the particle. The constant 8 in front is the hardsphere contribution. The value of $k_{\mathrm{D}}^{\prime}$ is given by (7)

$$
k_{\mathrm{D}}^{\prime}=k_{\mathrm{I}}^{\prime}-k_{\mathrm{f}}^{\prime},
$$

where $k_{\mathrm{f}}^{\prime}$ accounts for the change in the friction coefficient with concentration. For our analysis we have used the results of Felderhof (13)

$$
\begin{aligned}
& k_{\mathrm{f}}^{\prime}=6.44+\int_{0}^{\infty} {\left[12(1+x)-15 / 8(1+x)^{-2}\right.} \\
&\left.+\frac{27}{64}(1+x)^{-4}+\frac{75}{64}(1+x)^{-5}\right] \\
& \quad \times\left[1-e^{-v(x) / k_{\mathrm{B}} T}\right] d x .
\end{aligned}
$$

A similar expression is also given by Batchelor (14). Thus if we know the interaction potential $V(x)$ we can calculate $k_{\mathrm{D}}^{\prime}$ and $k_{\mathrm{I}}^{\prime}$.

According to the DLVO theory (15), $V(x)$ is the sum of an attractive part $V_{\mathrm{A}}$ and a repulsive part $V_{\mathrm{R}}$. The attractive part is due to London-van der Waals interaction and is given by Eq. [14] for the case of two spheres (16):

$$
\begin{aligned}
V_{A}= & -\left(\frac{A}{12}\right)\left[\left(x^{2}+2 x\right)^{-1}+\left(x^{2}+2 x+1\right)^{-1}\right. \\
& \left.+2 \ln \left(x^{2}+2 x\right) /\left(x^{2}+2 x+1\right)\right] ; \quad \text { [14] }
\end{aligned}
$$

$A$ is the Hamaker constant. The repulsive interaction is due to the electric charge of the spheres and their double layers; $V_{R}$ was approximated by (17)

$$
V_{\mathrm{R}}=\left(\epsilon a \psi_{0}^{2} / 2\right) \ln (1+\exp (-2 \kappa a x)),
$$

where $\epsilon$ is the dielectric constant of the suspending medium, $\psi_{0}$ is the potential of the diffuse layer surrounding the sphere, and $\kappa$ is the reciprocal Debye length given by

$$
\kappa^{2}=8 \pi C_{\mathrm{NaCl}} e^{2} Z^{2} /\left(\epsilon k_{\mathrm{B}} T\right),
$$

where $C_{\mathrm{NaCl}}$ is the concentration of the ions in solution and $Z$ is the valence of the ions. Equation [15] is valid for $k a \gg 1$. This form was used because $2 \leq \kappa a \leq 20$ for our solutions. The diffuse layer potential $\psi_{0}$ is further related to the micellar charge qe through (18)

$$
\begin{aligned}
\psi_{0}=\left(2 k_{\mathrm{B}} T / e\right) & \sinh ^{-1} \\
\times & {\left[2 \pi e^{-1} q e /\left(4 \pi a^{2} \epsilon k_{\mathrm{B}} T\right)\right] . }
\end{aligned}
$$

To prevent the divergence of the attractive part of the potential we used a lower cutoff of $X_{\mathrm{L}}=8 \times 10^{-2}$ corresponding to a closest approach of $4 \AA$. If $X_{\mathrm{L}}$ is decreased by two, the Hamaker constant $A$ is decreased by $40 \%$.

\section{RESULTS}

\section{A. Critical Micellar Temperatures}

The CMT values of solutions containing $0.07 M$ SDBS are shown as a function of salt concentration in Fig. 1a. For comparison the CMT values of $0.069 \mathrm{M}$ SDS taken from Ref. (3) are also shown. We see that the CMT values for SDBS solutions increase by $40^{\circ} \mathrm{C}$ in going from no salt to $0.5 \mathrm{M}$ salt. Critical micellar temperatures of SDS solutions, on the other hand, only increase by $\approx 8^{\circ} \mathrm{C}$ over the same range. Perhaps more important than the range is the difference in curvature between the two systems. The SDBS curve is concave up and the SDS curve is concave down with a continuously decreasing slope indicating that perhaps a limiting CMT exists for SDS but not for SDBS. The higher CMT values for SDBS are expected in that SDBS 


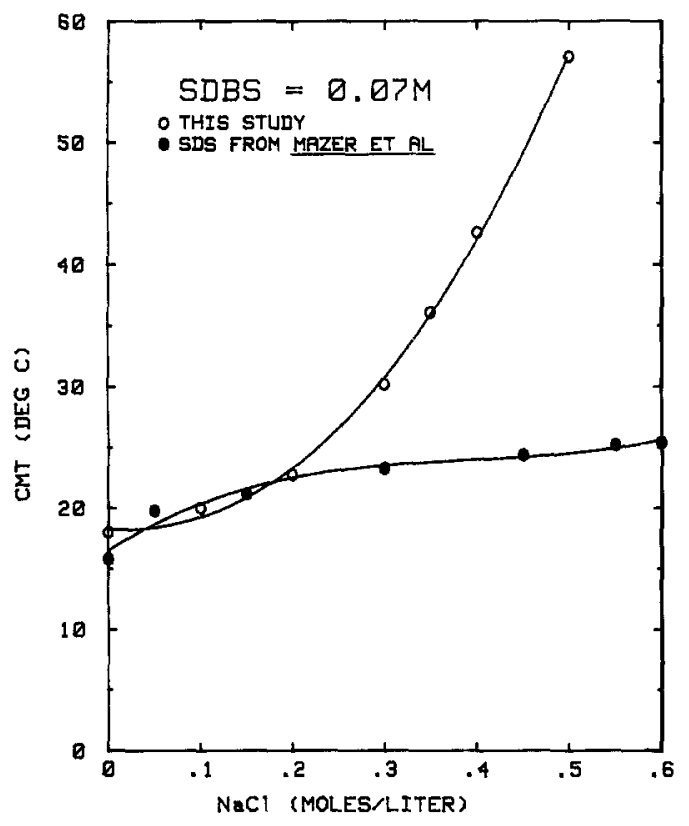

FIG. 1a. Plot of CMT values as a function of salt concentration for $0.07 M$ SDBS solutions. For comparison data for SDS are also shown.

has a larger hydrophobic part and the sulfonate group is less polar than the sulfate group of SDS. At this point we are not sure

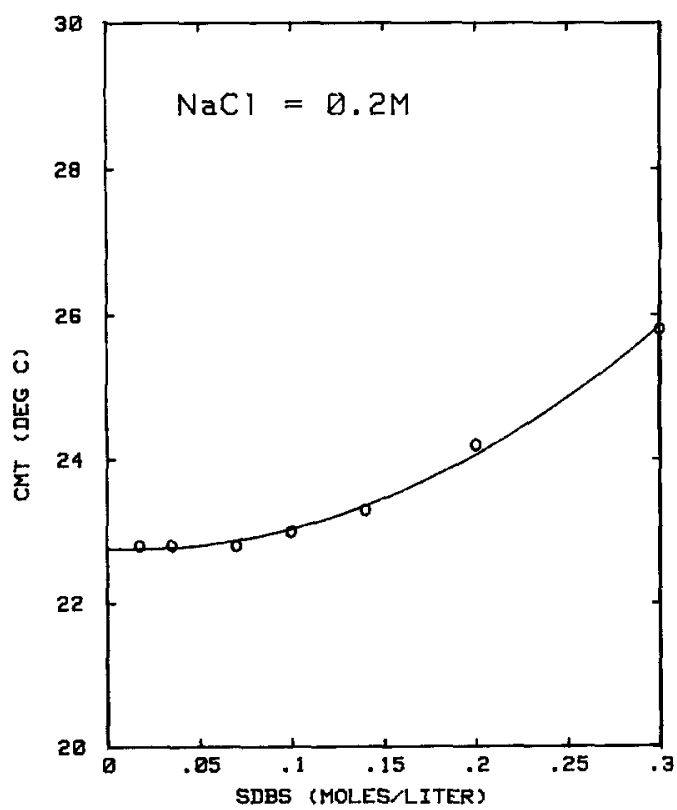

Fig. 1b. Dependence of CMT values on SDBS concentration for $0.2 \mathrm{M} \mathrm{NaCl}$ solutions. whether or not the presence of two isomers affects the shape of the CMT curve.

The CMT values of solutions containing $0.2 \mathrm{M} \mathrm{NaCl}$ are plotted as a function of SDBS concentration in Fig. 1b. While the shape of the curve is the same as that of Fig. $1 \mathrm{a}$, the increase is much smaller, only $4^{\circ} \mathrm{C}$ in going from 0 to $0.3 \mathrm{M}$ SDBS.

\section{B. Apparent Molecular Weights, Aggregation Numbers, and Radii of Gyration}

The scattered intensity was measured as a function of temperature, and salt and SDBS concentration. The apparent molecular weights and the aggregation numbers $\bar{n}$ were determined from the extrapolated zero angle intensity using Eq. [1]. Values of $\bar{n}$ determined in this fashion are shown on the righthand side vertical axes of Figs. 2 and 3, together with the corresponding $\bar{R}_{\mathrm{h}}$ values. The

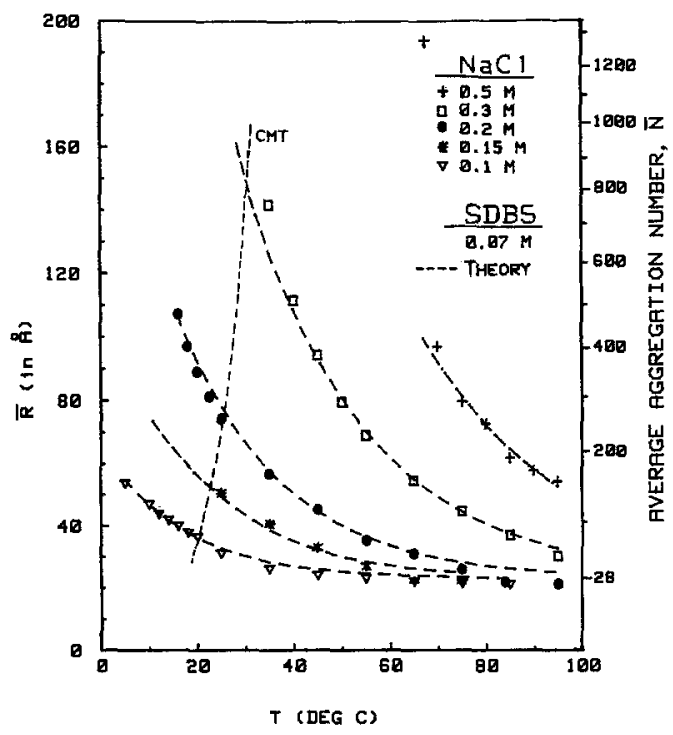

FIG. 2. Dependence of the Z-average hydrodynamic radius and the aggregation number on salt concentration and temperature for $0.07 M$ SDBS solutions. The aggregation numbers and the hydrodynamic radii were independently determined from intensity and correlation function measurements. The agreement between the two methods is less than the size of the data points. The dashed lines represent the predictions of the thermodynamic model described in the text. 


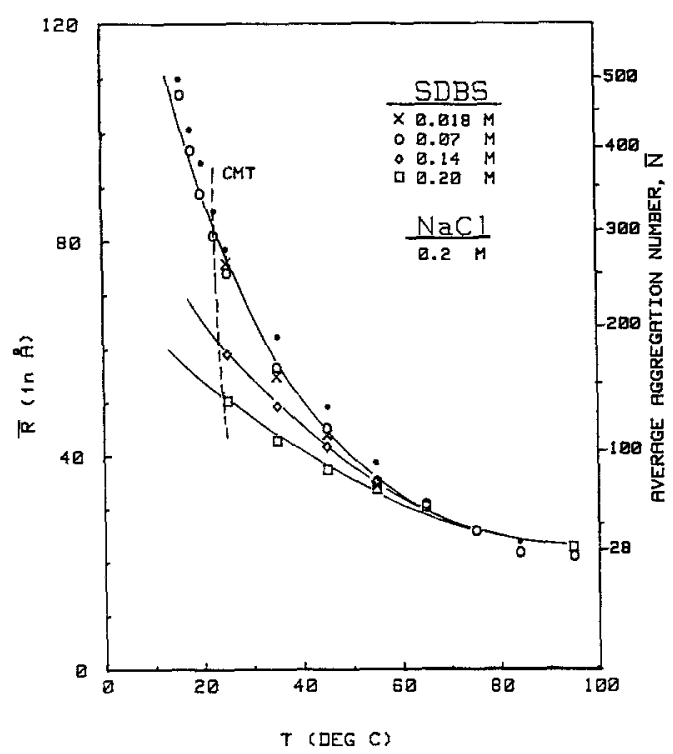

FIG. 3. Dependence of $\bar{R}_{\mathrm{h}}$ on SDBS concentration and temperature for $0.2 \mathrm{M} \mathrm{NaCl}$ solutions. The solid points are for independent determination of $n$ for $0.07 M$ SDBS solutions.

average aggregation number $\bar{n}$ ranged from a minimum of $\bar{n}=28$ to $\bar{n} \cong 1300$ for the solutions we investigated.

From Figs. 2 and 3 we see that as the temperature increases $\bar{n}$ decreases and reaches a concentration and temperature independent value of 28 . We took this to be the aggregation number $n_{0}$ of the minimum spherical micelle; $n_{0}=28$ is small compared to a minimum aggregation number of $60-80(7,10)$ for SDS but comparable to the aggregation numbers of other alkylaryl sulfonates, which

TABLE I

Radii of Gyration Data for $0.07 M$ SDBS

\begin{tabular}{|c|c|c|c|}
\hline \multirow[b]{2}{*}{$\begin{array}{l}C_{\mathrm{NaCC}} \\
(M)\end{array}$} & \multirow[b]{2}{*}{$\begin{array}{l}\text { Temp. } \\
\left({ }^{\circ} \mathrm{C}\right)\end{array}$} & \multicolumn{2}{|c|}{$\tilde{R}_{\mathbb{B}}(\AA)$} \\
\hline & & Expt1. & Theor. \\
\hline \multirow[t]{3}{*}{0.2} & 16 & 203 & 224 \\
\hline & 18 & 186 & 195 \\
\hline & 20 & 178 & 173 \\
\hline \multirow[t]{3}{*}{0.3} & 35 & 234 & 270 \\
\hline & 40 & 199 & 218 \\
\hline & 45 & 185 & 195 \\
\hline
\end{tabular}

TABLE II

Average Diffusion Coefficients and Variances in 0.07 $M$ SDBS as a Function of $\mathrm{NaCl}$ Concentration and Temperature

\begin{tabular}{|c|c|c|c|c|}
\hline \multirow[b]{2}{*}{$\begin{array}{l}C_{\mathrm{NaCl}} \\
(M) \\
\end{array}$} & \multirow{2}{*}{$\begin{array}{c}\text { Temp. } \\
\left({ }^{\circ} \mathrm{C}\right)\end{array}$} & \multirow{2}{*}{$\begin{array}{c}\text { Average } \\
\text { diffusion } \\
\text { coefficient } \\
\bar{D}\left(10^{-7} \mathrm{~cm}^{2} / \mathrm{sec}\right)\end{array}$} & \multicolumn{2}{|c|}{$\begin{array}{c}\text { Relative variance, } \\
V(\%)\end{array}$} \\
\hline & & & Exptl. & Theor. \\
\hline \multirow[t]{14}{*}{0.1} & 5.4 & 2.55 & 4.4 & 3.6 \\
\hline & 10 & 3.35 & 5.8 & 2.2 \\
\hline & 12 & 3.82 & 6.8 & 1.7 \\
\hline & 14 & 4.25 & 6.3 & 1.4 \\
\hline & 16 & 4.72 & 6.8 & 1.0 \\
\hline & 18 & 5.29 & 6.8 & 1.0 \\
\hline & 20 & 5.82 & 5.3 & .64 \\
\hline & 25 & 7.77 & 3.2 & 25 \\
\hline & 35 & 11.8 & 4.4 & .04 \\
\hline & 45 & 16.0 & 4.8 & .01 \\
\hline & 55 & 21.3 & 5.8 & .01 \\
\hline & 65 & 25.3 & 6.3 & .01 \\
\hline & 75 & 30.4 & 6.8 & .01 \\
\hline & 85 & 36.2 & 5.3 & .01 \\
\hline \multirow[t]{6}{*}{0.15} & 25 & 4.77 & 3.2 & 2.9 \\
\hline & 35 & 7.59 & 2.6 & 1.2 \\
\hline & 45 & 11.7 & 1.7 & 0.36 \\
\hline & 55 & 17.2 & 1.2 & 0.04 \\
\hline & 65 & 24.7 & 1.7 & 0.01 \\
\hline & 75 & 33.8 & 0.8 & 0.01 \\
\hline \multirow[t]{12}{*}{0.2} & 16 & 1.75 & 14 & 14.4 \\
\hline & 18 & 2.05 & 12 & 12.2 \\
\hline & 20 & 2.37 & 11 & 10.9 \\
\hline & 22.5 & 2.78 & 7.8 & 9.0 \\
\hline & 25 & 3.25 & 12 & 7.8 \\
\hline & 35 & 5.42 & 3.6 & 4.0 \\
\hline & 45 & 8.46 & 2.9 & 1.96 \\
\hline & 55 & 13.2 & 4.8 & 0.64 \\
\hline & 65 & 18.0 & 4.0 & 0.25 \\
\hline & 75 & 25.4 & 2.7 & 0.04 \\
\hline & 84 & 34.4 & 3.2 & 0.01 \\
\hline & 95 & 41.3 & 0.64 & 0.01 \\
\hline \multirow{9}{*}{0.3} & 35 & 2.54 & 28 & 18 \\
\hline & 40 & 3.06 & 18 & 16 \\
\hline & 45 & 4.01 & 12 & 12 \\
\hline & 50 & 5.26 & 7 & 7 \\
\hline & 55 & 6.67 & 3 & 7 \\
\hline & 65 & 10.1 & 2 & 3 \\
\hline & 75 & 14.5 & 2 & 2 \\
\hline & 85 & 20.4 & 1 & 1 \\
\hline & 95 & 28.9 & 1.2 & 0.2 \\
\hline \multirow[t]{8}{*}{0.5} & 65 & 2.61 & 12 & 12 \\
\hline & 67 & 3.05 & 6.2 & 8.4 \\
\hline & 70 & 6.05 & 3.2 & 7.3 \\
\hline & 75 & 7.94 & 2.3 & 4.8 \\
\hline & 80 & 9.42 & 1.7 & 4.0 \\
\hline & 85 & 11.9 & 0.5 & 3.2 \\
\hline & 90 & 13.7 & 0.3 & 2.0 \\
\hline & 95 & 15.6 & 0.2 & 0.8 \\
\hline
\end{tabular}


are in the range of 20 to $30(19 a, b, c)$. The difference between SDBS and SDS can be explained in terms of the differences in the structure of the hydrophobic parts and polarities of the head groups.

When the dissymmetry of the scattered intensity was high enough to determine reliable $R_{\mathrm{g}}$ values Eq. [2] was used to calculate the experimental $R_{\mathrm{g}}$ values for those samples. The experimental $\boldsymbol{R}_{\mathrm{g}}$ values, along with the calculated values using the thermodynamic model described above, are given in Table I.

\section{Average Diffusion Coefficients and Variances}

The values for the $Z$-average diffusion coefficient $\bar{D}$ and the relative variance $V$ obtained from dynamic light-scattering measurements are listed in Tables II and III. $\bar{D}$ was reproducible to $1 \%$ for the same sample and $3 \%$ for different samples under identical conditions. The corresponding percentages for $V$ were 3 and $5 \%$. Both $\bar{D}$ and $V$ are strong functions of temperature. From Table II we see that $\bar{D}$ increases with temperature at constant $\mathrm{NaCl}$ concentration and decreases with

\section{TABLE III}

Average Diffusion Coefficients and Variances in $\mathbf{0 . 2}$ $M \mathrm{NaCl}$ as a Function of SDBS Concentration and Temperature

\begin{tabular}{|c|c|c|c|c|}
\hline \multirow{2}{*}{$\begin{array}{l}C_{\mathrm{SDBS}} \\
(M)\end{array}$} & \multirow{2}{*}{$\begin{array}{l}\text { Temp. } \\
\left({ }^{\circ} \mathrm{C}\right)\end{array}$} & \multirow{2}{*}{$\begin{array}{l}\text { Average } \\
\text { diffusion } \\
\text { coefficient, } \\
\bar{D}\left(10^{-7} \mathrm{~cm}^{2} / \mathrm{sec}\right)\end{array}$} & \multicolumn{2}{|c|}{$\begin{array}{l}\text { Relative variance, } V \\
(\%)\end{array}$} \\
\hline & & & Exptl. & Theor. \\
\hline \multirow[t]{4}{*}{0.018} & 25 & 3.23 & 2.9 & 7.8 \\
\hline & 35 & 5.49 & 2.2 & 3.2 \\
\hline & 45 & 8.48 & 1.0 & 1.4 \\
\hline & 55 & 13.2 & 1.4 & 0.36 \\
\hline \multirow[t]{4}{*}{0.14} & 25 & 4.07 & 10 & 4.4 \\
\hline & 35 & 6.22 & 5.3 & 2.6 \\
\hline & 45 & 9.3 & 3.6 & 1.4 \\
\hline & 55 & 13.3 & 2.9 & 1.0 \\
\hline \multirow[t]{6}{*}{0.20} & 25 & 4.78 & 9.6 & 2.9 \\
\hline & 35 & 7.18 & 3.2 & 1.4 \\
\hline & 45 & 10.2 & 3.2 & 0.8 \\
\hline & 55 & 13.7 & 2.2 & 0.4 \\
\hline & 65 & 18.3 & 2.6 & 0.2 \\
\hline & 95 & 38.3 & 0.5 & 0.01 \\
\hline
\end{tabular}

increasing $\mathrm{NaCl}$ concentration at constant temperature. Variances decrease significantly with increasing temperature for high $\mathrm{NaCl}$ and SDBS concentrations but show no appreciable dependence of any of the variables for low-concentration solutions.

Values of $\bar{D}$ and $V$ for $0.2 \mathrm{M} \mathrm{NaCl}$ solutions are listed as a function of temperature and SDBS concentration in Table III; $\bar{D}$ increases with temperature and SDBS concentration. The relative variance decreases with temperature going from 10 to $0.5 \%$ for 0.14 and $0.2 M$ SDBS solutions and from 3 to $1.5 \%$ for $0.018 M$ SDBS solution.

\section{Apparent Hydrodynamic Radii}

The $Z$-average hydrodynamic radius $\bar{R}_{\mathrm{h}}$ calculated from Eq. [3] using the $\bar{D}$ values of Tables II and III are plotted in Fig. 2 as a function of temperature and $\mathrm{NaCl}$ concentration for $0.07 M$ SDBS solutions. Figure 3 shows the temperature and SDBS concentration dependence of $\bar{R}_{\mathrm{h}}$ for $0.2 \mathrm{M} \mathrm{NaCl}$ solutions. From Fig. 2 we see that $\bar{R}_{\mathrm{h}}$ varies dramatically with temperature and salt concentration. At high temperatures $\bar{R}_{\mathrm{h}}$ asymptotically approaches a minimum value of $\bar{R}_{\mathrm{h}}$ $=22 \AA$, which is independent of $\mathrm{NaCl}$ and SDBS concentration. We took this value to be the effective hydrodynamic radius of a minimum spherical SDBS micelle. The value $\bar{R}_{\mathrm{h}}=22 \AA$ is slightly less than the $25 \AA$ reported for the minimum SDS micelle (3) but in good agreement with what one would expect from adding up the bond lengths. As the temperature is lowered $\bar{R}_{\mathrm{h}}$ increases dramatically for solutions with $\mathrm{NaCl}$ concentrations greater than $0.15 M$ (roughly twice the SDBS concentration). At low $\mathrm{NaCl}$ concentrations only a moderate increase in $\bar{R}_{\mathrm{h}}$ occurs as the temperature is lowered. From Fig. 3 we see that for SDBS concentrations below $0.07 \mathrm{M}$, $\bar{R}_{h}$ is independent of SDBS concentration and decreases appreciably for higher SDBS concentrations. This decrease, however, is not a decrease in the real hydrodynamic radius. It is a decrease in the apparent hydrodynamic radius due to application of the Stokes-Einstein relationship when it should 
not be used (i.e., when there are strong charge interactions between the micelles). Figure 3 clearly demonstrates that when the salt-tosurfactant concentration ratio is at least two there is enough counterion shielding, and the micelle size is independent of surfactant concentration. When the ratio is less than two there is inadequate shielding and one should not use the Stokes-Einstein relationship to obtain the hydrodynamic size. The smoothness of the $\bar{R}_{\mathrm{h}}$ curves as they pass through the CMT points shows that the micellar size in the supercooled region has the same temperature dependence on $\mathrm{NaCl}$ concentration as above the CMT. Also the temperature dependence of the rate of increase is too slow compared to the power law. This behavior rules out the possibility of using critical phenomena theories to interpret micelle growth as the CMT is approached.

\section{E. Results of the Thermodynamic Model}

To use the thermodynamic model of micelle formation we first determined the temperature and salt concentration dependence of the parameter $K$ using the experimental

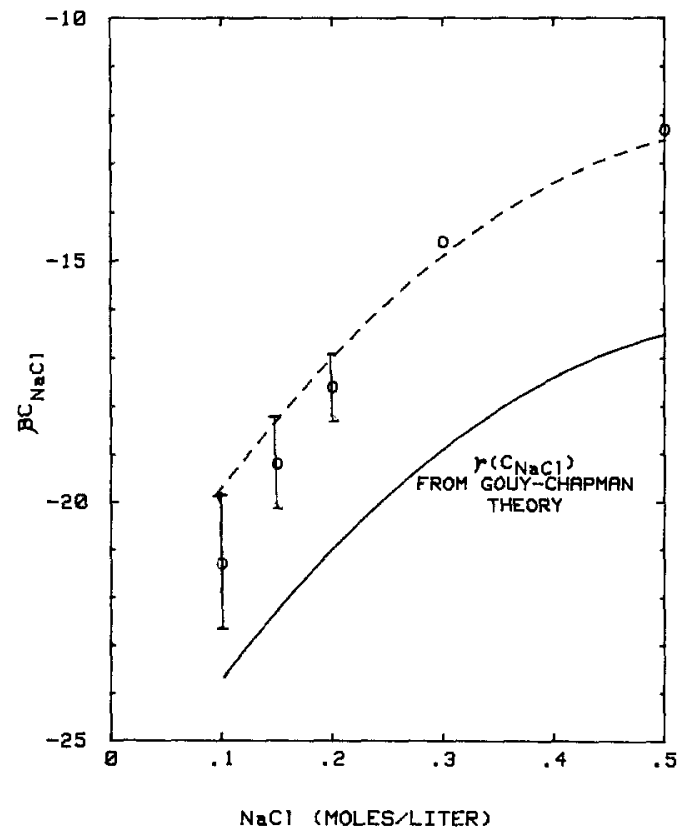

FIG. 4. Dependence of the thermodynamic model parameter $\beta\left(C_{\mathrm{NaCl}}\right)$ on salt concentration. data given in Fig. 2. The data for $\ln K$ can be represented by (10)

$$
\ln K\left(T, C_{\mathrm{NaCl}}\right)=\alpha / T+\beta\left(C_{\mathrm{NaCl}}\right) \text {. }
$$

For SDBS we determined $\alpha$ to be $10,470^{\circ} \mathrm{K}$ compared to $17,110^{\circ} \mathrm{K}$ found for SDS (10), and $\beta\left(C_{\mathrm{NaCl}}\right)$ is shown in Fig. 4. Theoretical values of $\bar{R}_{\mathrm{g}}$ calculated using the above values of $\alpha$ and $\beta$ are shown in Table I. Theoretical curves of $\bar{R}_{\mathrm{h}}$ calculated are shown in Fig. 2. We see that the agreement between the calculated and experimental $\bar{R}_{\mathrm{g}}$ and $\bar{R}_{\mathrm{h}}$ data is very good. One major problem with using the thermodynamic model with only lightscattering data is that it has two adjustable parameters $(\alpha$ and $\beta)$. Due to those two adjustable parameters the model can fit data (such as $0.1 \mathrm{M} \mathrm{NaCl}$ curve in Fig. 2) for which the Stokes-Einstein relationship does not hold. The calculated relative variances are also given in Tables II and III along with the experimentally measured values. The agreement between the calculated and observed variances is good for high salt concentrations but poor for low salt concentrations. This is not surprising since experimentally measured variances are one order of magnitude less accurate than $D$ values and are also affected by the contributions from electrostatic interactions (precisely the case for low salt solutions).

From the values of $\alpha$ and $\beta$ one can calculate the enthalpy $\Delta H$ and the entropy $\Delta S$ of the hydrophobic interaction (per $\AA^{2}$ ) of the hydrocarbon-water interface. For SDBS they are $\Delta H=18.9 \mathrm{cal} / \mathrm{mole} / \AA^{2}$ and $\Delta S$ $=-7.2 \times 10^{-3} \mathrm{cal} / \mathrm{mole} /{ }^{\circ} \mathrm{K} / \AA^{2}$. The corresponding values for SDS determined by Mazer et al. (10) are $\Delta H=22.4 \mathrm{cal} / \mathrm{mole} / \AA^{2}$ and $\Delta S=-5.9 \times 10^{-3} \mathrm{cal} / \mathrm{mole} /{ }^{\circ} \mathrm{K} / \AA^{2}$.

\section{F. Results of the Intermicellar Interaction Model}

To determine how $k_{\mathrm{D}}^{\prime}$ and $k_{\mathrm{I}}^{\prime}$ depend on temperature, and SDBS and $\mathrm{NaCl}$ concentration extensive measurements of the apparent molecular weight and diffusion coefficient were made; $k_{\mathrm{D}}^{\prime}$ and $k_{\mathrm{I}}^{\prime}$ are related to $k_{\mathrm{D}}$ and $k_{\mathrm{I}}$ by $k_{\mathrm{D}}^{\prime}=k_{\mathrm{D}} \bar{v}$ and $k_{\mathrm{I}}^{\prime}=k_{\mathrm{I}} \bar{v}, \bar{v}$ is the 


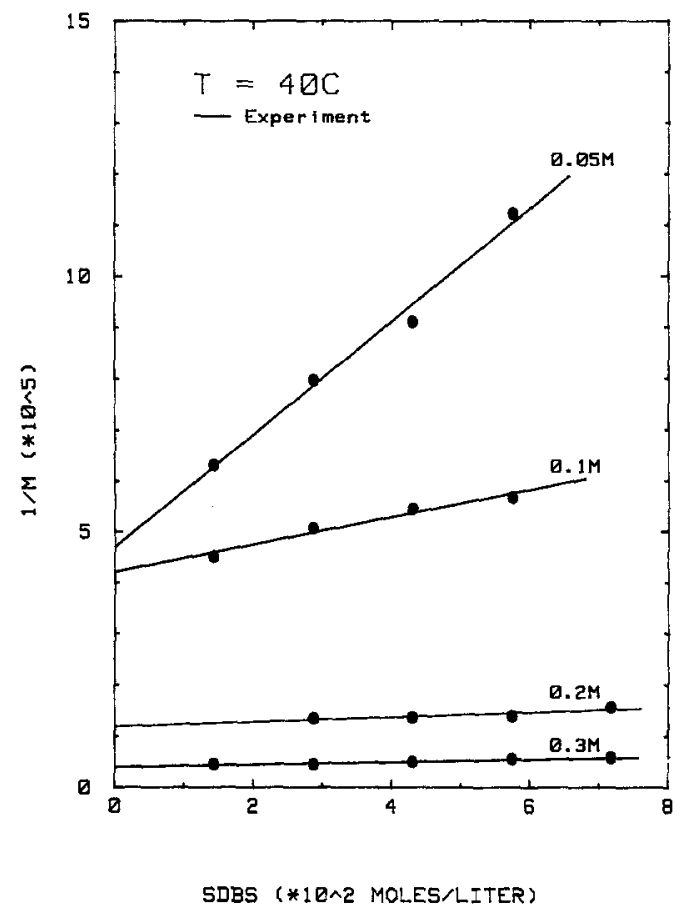

FIG. 5a. Plot of apparent molecular weight $M^{-1}$ as a function of SDBS and $\mathrm{NaCl}$ concentration at $40^{\circ} \mathrm{C}$.

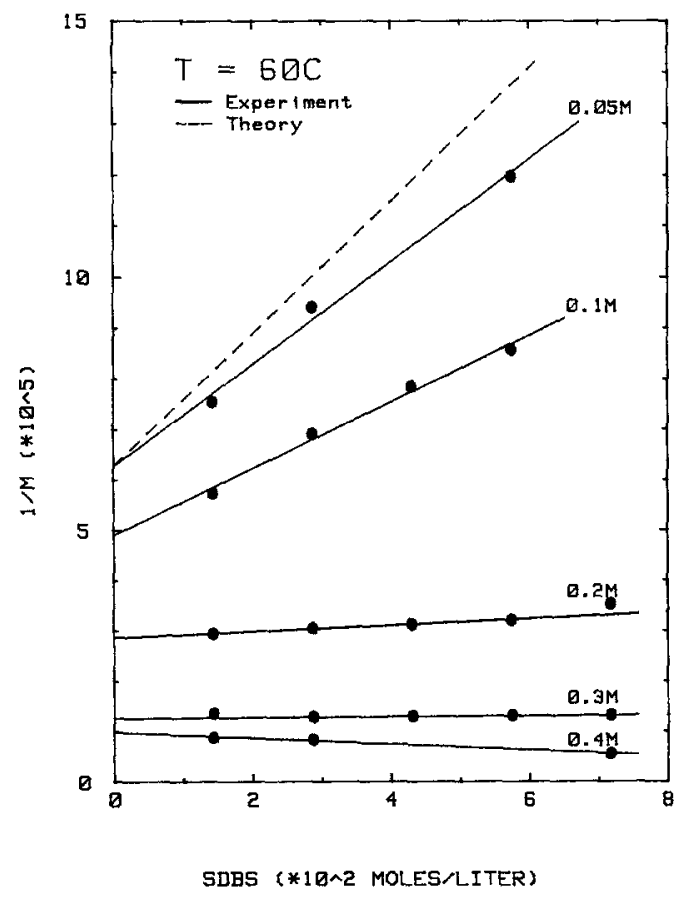

FIG. 5b. Plot of apparent molecular weight $M^{-1}$ as a function of SDBS and $\mathrm{NaCl}$ concentration at $60^{\circ} \mathrm{C}$. The dashed line is the best fit theoretical curve.
TABLE IV

Scattered Intensity Data at $40^{\circ} \mathrm{C}$

\begin{tabular}{llllc}
\hline & & & \multicolumn{2}{c}{$K_{\mathrm{I}}(1 / M)$} \\
\cline { 4 - 5 } $\begin{array}{c}C_{\text {NaC }} \\
(M)\end{array}$ & $10^{\mathrm{s} M_{0}^{-1}}$ & \multicolumn{1}{c}{$n$} & \multicolumn{1}{c}{ Exptl. } & Theor. \\
\hline 0.05 & 4.7 & 61 & 23.51 & 19.38 \\
0.1 & 4.21 & 68 & 6.42 & 10.79 \\
0.2 & 1.2 & 228 & 3.74 & 8.3 \\
0.3 & 0.42 & 683 & 0.01 & 4.24 \\
\hline
\end{tabular}

specific volume of the micelle. The plots of $M^{-1}$ are shown in Figs. 5a and 5b. The data points were fitted with $1 / M=1 / M\left(1+k_{\mathrm{I}}(c\right.$ $\left.-c_{0}\right)$ ) to obtain the experimental values of $M_{0}$ and $k_{\mathrm{I}}$ reported in Tables IV and V. From Table IV we see that the aggregation number at the CMC varies from a minimum of 61 for $0.05 \mathrm{M} \mathrm{NaCl}$ to a maximum of $\sim 680$ for the $0.3 \mathrm{M} \mathrm{NaCl}$ solution at $40^{\circ} \mathrm{C}$. For $0.3 \mathrm{M}$ $\mathrm{NaCl}$ solutions $k_{\mathrm{I}}$ is zero. At $60^{\circ} \mathrm{C}$ the aggregation number at the $\mathrm{CMC}$ varies from 46 to 226 for the same salt concentrations and again $k_{\mathrm{I}}$ is approximately zero for 0.32 $M \mathrm{NaCl}$ solutions. The dependence of the aggregation number at the $\mathrm{CMC}$ on the salt concentration is much stronger than its dependence on the temperature.

The apparent diffusion coefficient, $D$, data are shown in Figs. 6a and 6b. The experimental data was fitted with $D=D_{0}\left(1+k_{\mathrm{D}}(c\right.$ $\left.-c_{0}\right)$ ) to determine the $D_{0}$ and $k_{\mathrm{D}}$ values as a function of temperature and salt concentration. The results are given in Tables VI and VII. The extrapolated diffusion coefficient $D_{0}$ increases markedly with decreasing salt concentration and temperature and de-

TABLE V

Scattered Intensity Data at $60^{\circ} \mathrm{C}$

\begin{tabular}{lcccr}
\hline & & & \multicolumn{2}{c}{$K_{\mathrm{I}}(\mathrm{l} / M)$} \\
\cline { 4 - 5 } $\begin{array}{l}C_{\text {NaCl }} \\
(M)\end{array}$ & $10^{5} M_{0}^{-1}$ & $n$ & \multicolumn{1}{c}{ Exptl. } & Theor. \\
\hline 0.05 & 6.28 & 46 & 16.01 & 20.71 \\
0.1 & 4.89 & 58 & 13.46 & 8.53 \\
0.2 & 2.86 & 100 & 2.2 & 5.85 \\
0.3 & 1.27 & 226 & 0.61 & 5.33 \\
0.4 & 0.99 & 290 & -5.93 & 1.96 \\
\hline
\end{tabular}




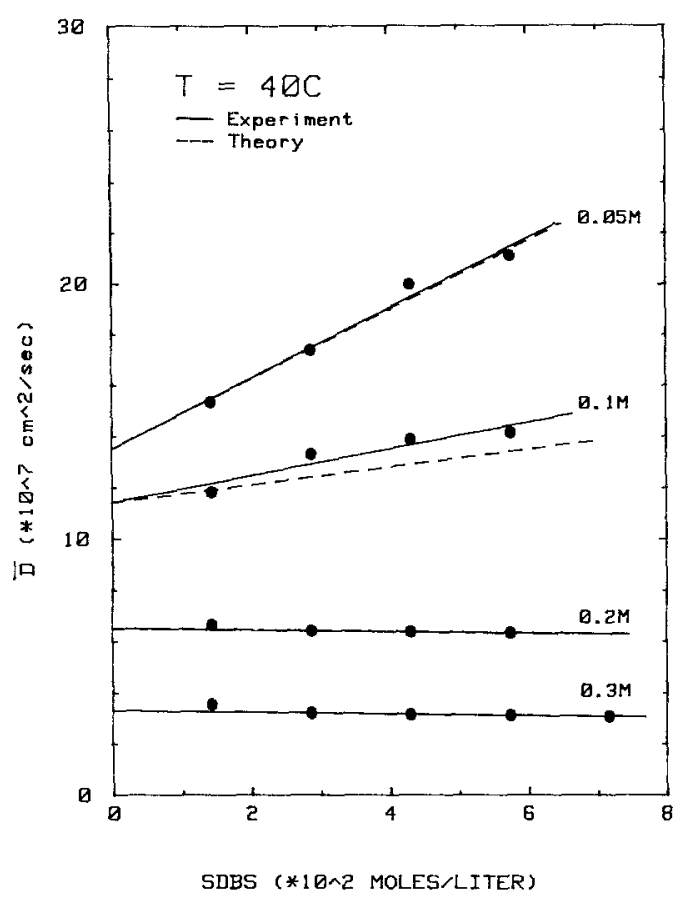

FIG. 6a. Dependence of the apparent diffusion coefficient on SDBS and $\mathrm{NaCl}$ concentration at $40^{\circ} \mathrm{C}$. The dashed lines are the best fit theoretical curves.

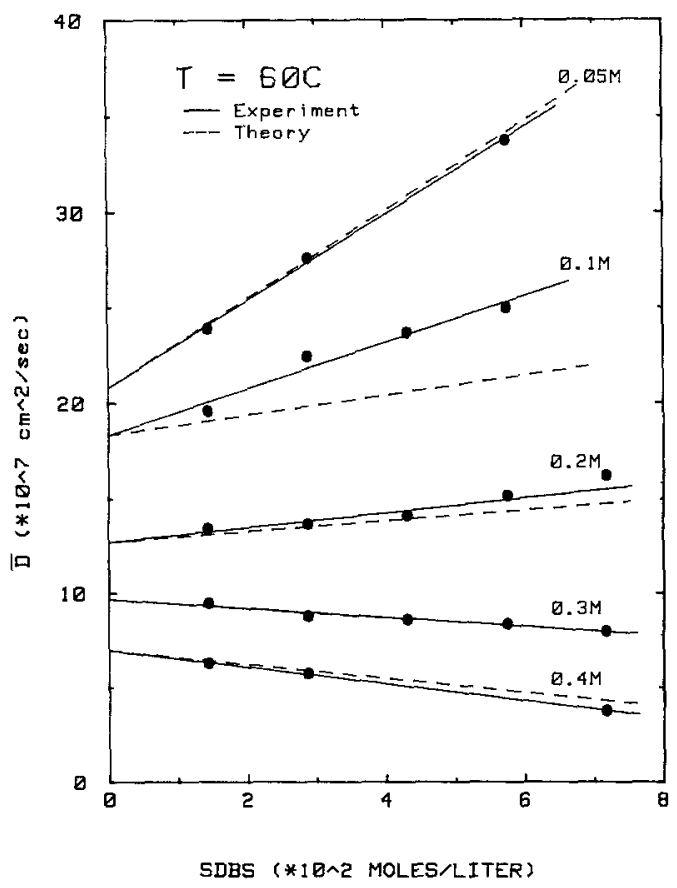

FIG. 6b. Dependence of the apparent diffusion coefficient on SDBS and $\mathrm{NaCl}$ concentration at $60^{\circ} \mathrm{C}$. The dashed lines are the best fit theoretical curves.
TABLE VI

Intensity Correlation Data at $40^{\circ} \mathrm{C}$

\begin{tabular}{lrrrr}
\hline & & & \multicolumn{2}{c}{$K_{\mathrm{D}}(1 / M)$} \\
\cline { 5 - 6 } $\begin{array}{l}C_{\text {Nad }} \\
(M)\end{array}$ & $\begin{array}{c}10^{7} D_{0} \\
\left(\mathrm{~cm}^{2} / \mathrm{sec}\right)\end{array}$ & \multicolumn{1}{c}{$\begin{array}{c}R_{\mathrm{h}} \\
(\AA)\end{array}$} & \multicolumn{1}{c}{ Exptl. } & Theor. \\
\hline 0.05 & 13.5 & 26 & 10.24 & 10.11 \\
0.1 & 11.4 & 30.8 & 4.58 & 3.05 \\
0.2 & 6.5 & 59.4 & -0.47 & -0.46 \\
0.3 & 3.3 & 106.1 & -0.99 & -1.16 \\
\hline
\end{tabular}

creases with increasing salt concentration. The slope $k_{\mathrm{D}}$ is a decreasing function of the salt concentration. The salt concentrations corresponding to zero slope were found by interpolation to be

$$
\begin{array}{rlll}
\text { at } 40^{\circ} \mathrm{C} k_{\mathrm{I}}=0 & \text { for } & 0.30 \mathrm{M} \mathrm{NaCl} \\
k_{\mathrm{D}}=0 & \text { for } & 0.18 \mathrm{M} \mathrm{NaCl}, \\
\text { at } 60^{\circ} \mathrm{C} k_{\mathrm{I}}=0 & \text { for } & 0.32 \mathrm{M} \mathrm{NaCl} \\
k_{\mathrm{D}}=0 & \text { for } & 0.25 \mathrm{M} \mathrm{NaCl} .
\end{array}
$$

In order to be able to compute the theoretical values of $k_{\mathrm{I}}$ and $k_{\mathrm{D}}$ given in Table IV through VII we assumed that the Hamaker constant $A$ and the micellar charge $q$ appearing in Eqs. [14] and [17] were both independent of the salt concentration. According to recent measurements $(20,21)$ both of these assumptions appear to be reasonable. One fundamental assumption involved in the derivation of Eqs. [11] and [13], that the interacting particles are spherical, is definitely not satisfied by the SDBS micelles at high salt concentrations. In $0.4 \mathrm{M} \mathrm{NaCl}$ solutions the aspect ratio of the micelles are as high as 10 .

TABLE VII

Intensity Correlation Data at $60^{\circ} \mathrm{C}$

\begin{tabular}{lccrr}
\hline & & & \multicolumn{2}{c}{$K_{\mathrm{D}}(1 / M)$} \\
\cline { 5 - 6 } $\begin{array}{c}C_{\text {Nad }} \\
(M)\end{array}$ & $\begin{array}{c}10^{7} D_{\mathrm{O}} \\
\left(\mathrm{cm}^{2} / \mathrm{sec}\right)\end{array}$ & $\begin{array}{c}R_{\mathrm{h}} \\
(\AA)\end{array}$ & \multicolumn{1}{c}{ Exptl. } & Theor. \\
\hline 0.05 & 20.8 & 25.2 & 10.92 & 11.19 \\
0.1 & 18.3 & 28.6 & 6.63 & 2.85 \\
0.2 & 12.4 & 41.1 & 3.07 & 2.25 \\
0.3 & 9.7 & 54.1 & -2.44 & -2.52 \\
0.4 & 7 & 74.9 & -6.33 & -5.29 \\
\hline
\end{tabular}


Best fit values of $A$ and $q$ at each temperature were determined by an iterative procedure. First for $0.05 \mathrm{M} \mathrm{NaCl}$ solutions we neglected the attractive part of $V(x)$ and obtained a $q$ value that reproduced the experimentally determined $k_{\mathrm{D}}$ values. At high salt concentrations $(0.3 \mathrm{M} \mathrm{NaCl})$ there is a balance between the attractive and the repulsive part of $V(x)$, the $q$ values determined above were then used to obtain the best value of the Hamaker constant that would reproduce the $k_{\mathrm{D}}$ values for $0.3 \mathrm{M} \mathrm{NaCl}$ solutions. This value of $A$ was substituted back into the determination of $q$ for $0.05 \mathrm{M} \mathrm{NaCl}$ solutions to get a better estimate of the actual $q$ which in turn was used to obtain a new $A$ value. The final "best" values are

$$
\begin{aligned}
\text { at } 40^{\circ} \mathrm{C} q=12, & A / k_{\mathrm{B}} T=3.6, \\
\text { at } 60^{\circ} \mathrm{C} q=10, & A / k_{\mathrm{B}} T=5.4 .
\end{aligned}
$$

These can be compared to $q=37$ for SDS and $A / k_{\mathrm{B}} T=11.3$. The lower $q$ value is to be expected since the SDBS critical micellar temperatures and micelle sizes are much stronger functions of salt concentrations. The above values of $q$ and $A$ probably have significant errors since our micelles deviate from a spherical shape, and also the assumption of salt concentration independent $q$ and $A$ may not be valid. Despite these oversimplifying assumptions the theoretical curves calculated using the best $q$ and $A$ values are in good agreement with the experimental data as shown in Figs. 5 and 6. Again like the thermodynamic model the good agreement may be due to the determination of $A$ and $q$ from the same data. Independent knowledge of $q$ (or $A$ ) would have made a more rigorous test of this approach.

\section{G. Results of Dilution Experiments and Evidence for Mixed Micellization}

Before starting this project one of our aims was to determine the axial ratio of the micelles by making simultaneous measurements of the translational diffusion coefficient $D$ and the rotational diffusion coefficient $\Theta_{R}$. We first tried obtaining $\theta_{R}$ from the autocorrelation function of the depolar- ized light. Our first attempt was with a 0.07 $M$ SDBS solution containing $0.3 M \mathrm{NaCl}$. Much to our surprise the intensity of the depolarized scattering was very small $(1-3 \%$ of the polarized scattering) and it was not possible to obtain any correlation functions of the depolarized light. A calculation of the center-to-center distance between the rodlike SDBS micelles yielded values that were significantly less than the length of the micelles. To eliminate this problem the solution was diluted in a stepwise fashion and the data shown in Figs. 7 and 8 were obtained. From these dilution measurements we had to conclude that we could never have freely rotating micelles. Figures 7 and 8 also show that below $10 \times 10^{-3} M$ SDBS the micelles start increasing in size with dilution. This can happen under two conditions: (i) when there is mixed micellization or (ii) where there are trace amounts of high molecular weight alcohol impurity in the surfactant. We believe the first mechanism is the probable reason for the formation of very large aggregates upon dilution. Since our surfactant consists of ortho and para isomers, the ortho isomer having the higher $\mathrm{CMC}$ in aqueous solution

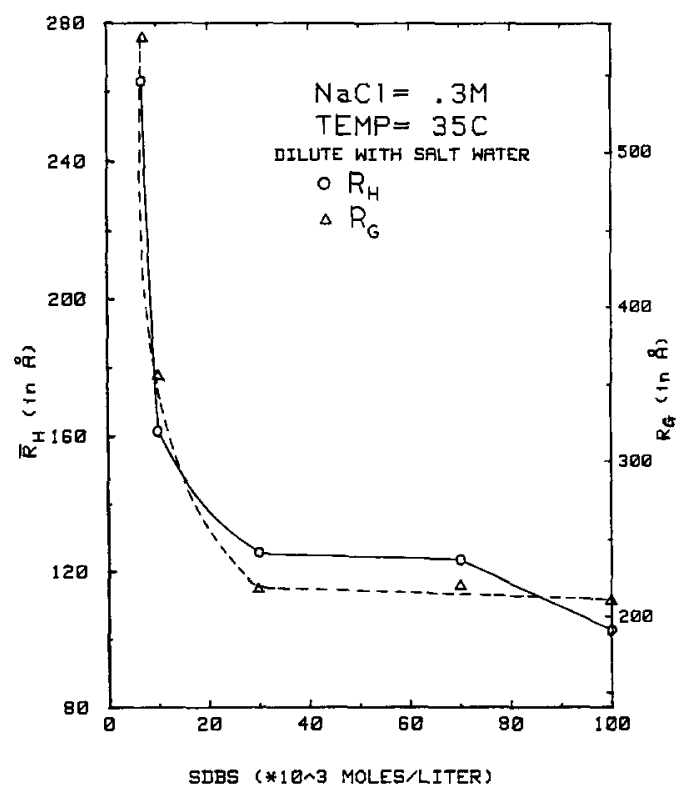

FIG. 7. Plot of $\bar{R}_{\mathrm{b}}$ and $\bar{R}_{\mathrm{g}}$ on SDBS concentration when diluted with $0.3 \mathrm{M} \mathrm{NaCl}$ salt solution. 


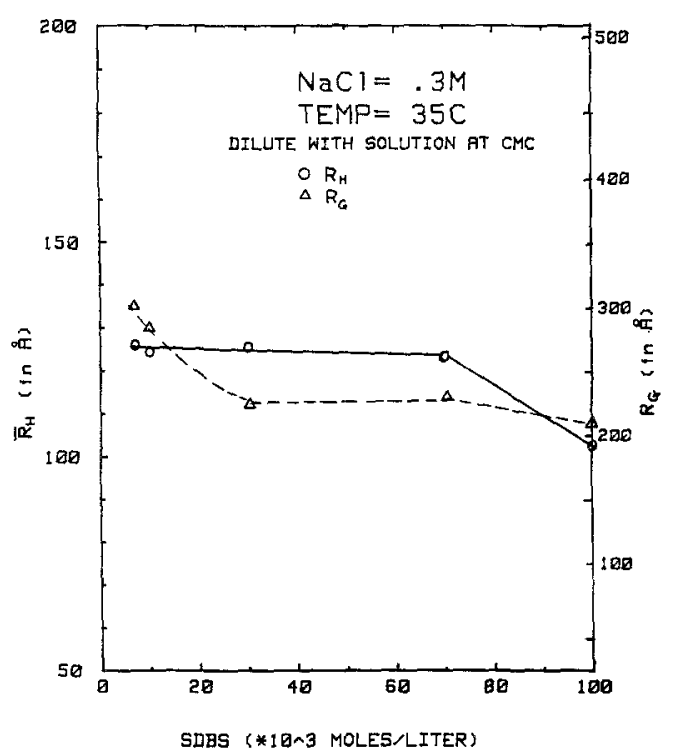

FIG. 8. Plot of $\bar{R}_{\mathrm{h}}$ and $\bar{R}_{\mathrm{g}}$ on SDBS concentration when dilution is achieved with a $0.3 \mathrm{M} \mathrm{NaCl}$ solution slightly below its $\mathrm{CMC}$.

is preferentially lost to the bulk fluid until there is not enough of it left in the micelles to keep the para isomer from forming large hydrated crystallites. Dilution with a solution approximately at the $\mathrm{CMC}$ extends the range over which micellar size remains constant but does not prevent the eventual formation of large crystallites as shown in Fig. 8. From Figs. 7 and 8 we see that there is no change in $\bar{n}$ and $\bar{R}_{\mathrm{h}}$ when the surfactant concentration is decreased from 0.07 to $0.03 \mathrm{M}$ or less. This indicates that for the majority of surfactant concentrations used in this study $\bar{R}_{\mathrm{h}}$ and $\bar{n}$ are independent of the surfactant concentration.

\section{H. The Shape of the SDBS Micelles}

If we assume that the minimum micelle is spherical in shape and has $\bar{R}_{\mathrm{h}}=22 \AA$ we can then combine the intensity information with the $\bar{R}_{\mathrm{h}}$ information and calculate the average scattered intensity by assuming the micelles to be spherical, prolate ellipsoids or oblate ellipsoids of revolution. The results of such calculations along with the experimental data are shown in Fig. 9. Clearly the data favor the prolate ellipsoid or the rod shape.
Figure 10 shows the excellent linearity between $n$ obtained from the apparent molecular weight measurements and $L$ the length of the rod-like micelles calculated from the radius, adding further support to the prolate ellipsoid model of the SDBS micelles.

\section{CONCLUSIONS}

We have deduced the size and shape of SDBS micelles as a function of temperature, and $\mathrm{NaCl}$ and SDBS concentration over a very wide range. We find that at high temperatures SDBS micelles approach a minimum size of $\bar{R}_{\mathrm{h}}=22 \AA$ and an aggregation number $\bar{n}=28$ independent of $\mathrm{NaCl}$ and SDBS concentration. Based on the assumption that the semiminor axis of the rod-like micelles is fixed as $22 \AA$, the semimajor axis of the micelles increases strongly with $\mathrm{NaCl}$ concentration as the temperature is lowered.

Using a thermodynamic model of micelle formation to analyze our data we find that there is very good agreement between the

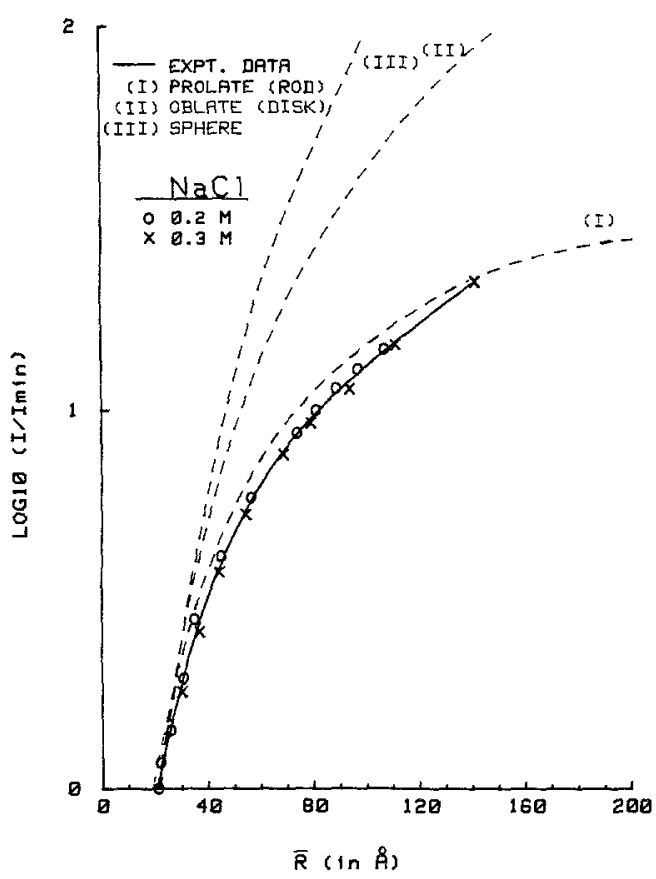

FIG. 9. Plot of the relative scattered intensity for SDBS solutions along with theoretically calculated curves for various geometrical shapes. 


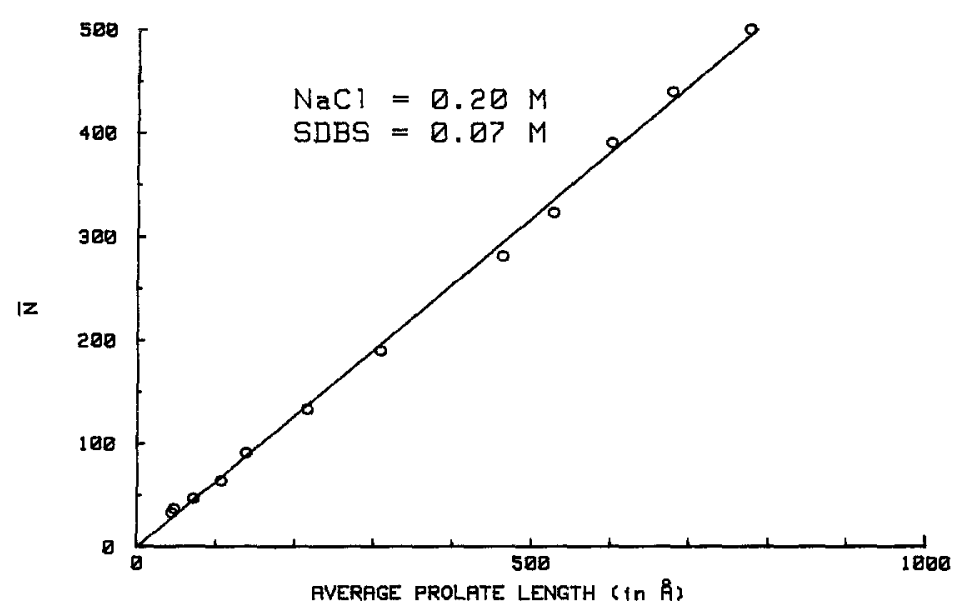

FIG. 10. Linear relationship between the aggregation number $n$ determined from intensity measurements and the length of the SDBS micelle $L$, calculated from $\bar{R}_{\mathrm{h}}$ measurements by dynamic light scattering. The slope indicates that in the cylindrical portion the length increases by $1.5 \AA$ for each SDBS molecule added to the micelle.

theoretical predictions of micelle size and the experimental measurements. We also used the DLVO theory of colloidal interactions to explain the salt concentration dependence of intermicellar interactions. From this analysis we determined the net charge on an SDBS micelle to be $\sim 10$ and the Hamaker constant to be $A \sim 5 k_{\mathrm{B}} T$. Both of these values are in qualitative agreement with those found for the SDS system. Due to the presence of two adjustable parameters both models can fit the experimental data quite well even in regions where they are not valid. The thermodynamic model is valid for very low surfactant concentrations of SDBS and salt concentrations that are at least twice that of the surfactant. Below the two-to-one ratio intermicellar charge interactions become important and an interacting micelle model based on the DLVO theory is more appropriate.

Dilution experiments close to the CMC indicate that due to a mixture of ortho and para isomers our surfactant forms mixed micelles.

One disturbing observation was the lack of depolarized scattering and our inability to determine a rotational diffusion coefficient. It remains to be seen whether this was due to the entanglement of long rod-like micelles or not. We hope to resolve this question by using an isomerically purer surfactant and other experimental techniques.

We are continuing our studies of SDBS solutions in aqueous and nonpolar solvents in order to obtain a better understanding of micelle formation, and will report our results in the near future.

\section{ACKNOWLEDGMENTS}

Support of this research by the Chemical and Process Engineering Division of the National Science Foundation under Grants ENG. 7910267 and CPE 7916518 is gratefully acknowledged.

\section{REFERENCES}

1. Anacker, E. W., in "Solution Chemistry of Surfactants" (K. L. Mittal, Ed.), Vol. 1, p. 247. Plenum, New York, 1979.

2. Emerson, M. F., and Holtzer, H., J. Phys. Chem. 71, 1898 (1967).

3. Mazer, N. A., Benedek, G. B., and Carey, M. C., J. Phys. Chem. 80, 1075 (1976).

4. Rohde, A., and Sackmann, E., J. Colloid Interface Sci. 70, 494 (1979).

5. Corti, M., and Degiorgio, V., Ann. Phys. Paris 3, 303 (1978).

6. Briggs, J., Nicoli, D. F., and Ciccolello, R., Chem. Phys. Lett. 73, 149 (1980).

7. Corti, M., and Degiorgio, V., J. Phys. Chem. 85, 711 (1981).

8. Gulari, E., Bedwell, B., and Alkhfaji, S., J. Colloid Interface Sci. 77, 202 (1980).

9. Koppel, D. E., J. Chem. Phys. 57, 4814 (1972). 
10. Mazer, N. A., Carey, M. C., and Benedek, G. B., in "Micellization, Solubilization and Microemulsions" (K. L. Mittal, Ed.), p. 359. Plenum, New York, 1977.

11. Tausk, R. J. M., and Overbeek, J. T. C., Biophys. Chem. 2, 175 (1974).

12. Pusey, P. N., in "Photon Correlation and Light Beating Spectroscopy" (H. Z. Cummins and E. R. Pike, Eds.), p. 387. Plenum, New York, 1975.

13. Felderhof, B. U., J. Phys. A. 11, 929 (1978).

14. Batchelor, G. K., J. Fluid Mech. 74, 1 (1976).

15. Verwey, E. J. W., and Overbeek, J. T. G., "Theory of the Stability of Lyophobic Colloids," Elsevier, New York, 1948.
16. Hamaker, H. C., Physica 4, 1058 (1937).

17. Hogg, R., Healy, T. W., and Fuerslenau, D. W., Trans. Faraday Soc. 62, 1638 (1966).

18. Anderson, J. L., Rauh, F., and Morales, A., J. Phys. Chem. 82, 608 (1978).

19. (a) Magid, L. J., Shaver, R. J., Gulari, E., Bedwell, B., and Alkhafaji, S., Preprints, ACS Division of Petroleum Chemistry, 26, 93 (1981). (b) Hoffmann, H., Ber. Bunsenges. Phys. Chem. 82, 988 (1978). (c) Yurzhenko, A. I., and Kucher, R. V., Dokl. Akad. Nauk. SSSR 85, 1337 (1952).

20. Israelachvili, J. N., and Adams, G. E., J. Chem. Soc. Faraday Trans. 1 74, 975 (1978).

21. Lindman, B., and Wennerstrom, H., Top. Curr. Chem. 87, (1979). 